\title{
Pour en lire plus : « Exploring the Gap »
}

\section{Barbara Bader}

\section{(2) OpenEdition}

\section{Journals}

Édition électronique

URL : http://journals.openedition.org/ere/5553

DOI : $10.4000 /$ ere. 5553

ISSN : 2561-2271

\section{Éditeur}

Centr'ERE

\section{Référence électronique}

Barbara Bader, « Pour en lire plus : «Exploring the Gap » "Éducation relative à l'environnement [En ligne] Volume 4 | 2003, mis en ligne le 14 septembre 2003, consulté le 24 septembre 2020. URL : http:// journals.openedition.org/ere/5553; DOI : https://doi.org/10.4000/ere.5553 


\title{
Pour en lire plus : « Exploring the Gap »
}

\author{
Barbara Bader
}

\section{RÉFÉRENCE}

Numéro spécial : « Exploring the Gap », Environmental Education Research, Volume 8, numéro 3, 2002.
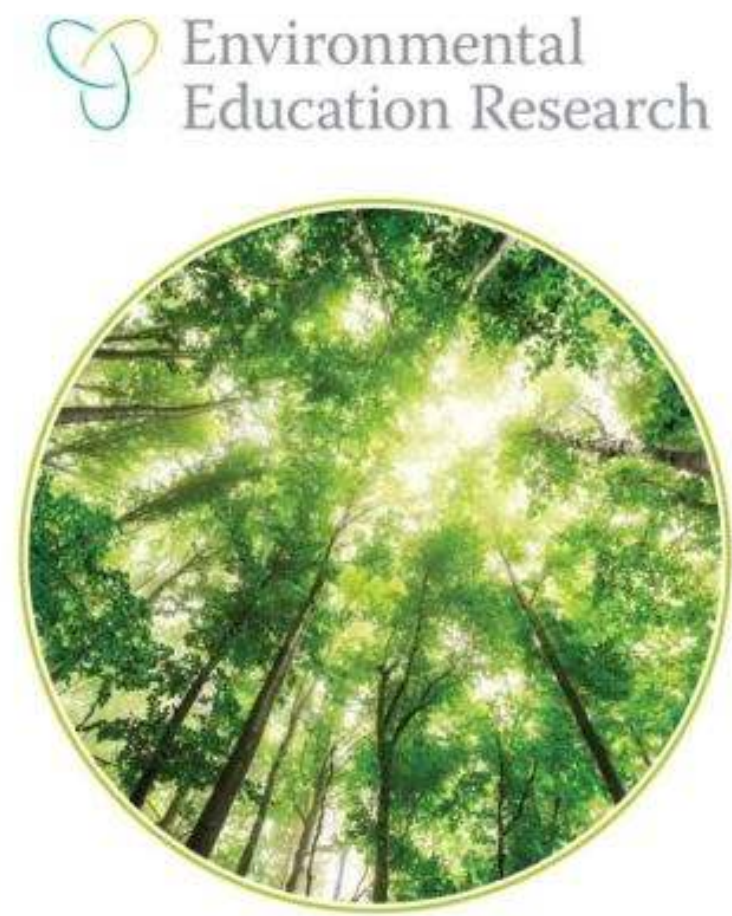
1 Ce numéro spécial de la revue Environmental Education Research est structuré autour de la mise en perspective d'un modèle comportementaliste de recherche en éducation relative à l'environnement (ERE). Ce modèle proposé dans le premier article par Anja Kollmuss et Julian Agyeman (2002) tente d'expliquer les raisons du « fossé » séparant l'acquisition de connaissances et la prise de conscience environnementale de l'engagement effectif dans des actions de protection de l'environnement. Les auteurs spécifient et relient entre eux certains «facteurs internes et externes» qui favoriseraient ou qui tendraient au contraire à inhiber ce que l'on nomme ici un comportement «pro-environnemental» en proposant une synthèse à partir de modèles antérieurs analogues. Cette manière de problématiser la recherche en ERE en termes de modifications de comportements se situe dans le prolongement d'un courant de recherche caractéristique des années 1980 qui a déjà été remis en question par ailleurs. Afin de poursuivre ce débat, les auteurs qui signent les sept autres textes de ce numéro proposent une mise en perspective critique et constructive de la position développée dans l'article initial de Kollmuss et Agyeman.

2 Illustrons quelques-uns des points de vue qui y sont développés. Soulignons tout d'abord, à la suite de Stephen Gough (2002), que la définition plutôt simpliste « d'un comportement pro-environnemental », essentiellement orienté vers l'atténuation de l'impact négatif de nos actions sur l'environnement naturel et bâti, proposée par Kollmuss et Agyeman mériterait d'être reconsidérée. Rob O'Donoghue et Heila LotzSisitka (2002) soulignent les limites d'une telle approche instrumentale tout en rappelant les critiques récurrentes se rapportant à ce type de conceptualisation que les auteurs ne semblent pas avoir prises en considération. Ils rappellent l'inscription sociohistorique d'une telle démarche qui reconduit implicitement certains rapports de pouvoir et illustrent leur propos à partir d'un exemple issu d'Afrique du Sud.

3 Pour sa part, Stephen Gough (2002) contextualise tout d'abord le sens qui peut être accordé à cette idée de conduite "pro-environnementale » et utilise des concepts provenant du répertoire de l'anthropologie culturelle pour reconsidérer le modèle comportementaliste. Gough soutient qu'en fonction des positions sociales des répondants, les rationalités des acteurs pourront être plurielles, leur définition d'un comportement pro-environnemental s'en trouvant modifiée selon les règles en vigueur dans le contexte en jeu (Gough et Scott, 1999). Il termine en soulignant ce qui a déjà été amplement illustré par ailleurs, à savoir que l'impulsion positiviste qui conduit à imaginer le chercheur comme entièrement détaché de son objet d'étude semble bien vivace dans la communauté des chercheurs en ERE mais manquerait de fécondité, ne permettant pas de rattacher les résultats de recherche aux contextes qui en éclairent le sens. Tout en soulignant que l'approche de Kollmuss et Agyeman pourrait avoir un intérêt si elle était jumelée à une approche plus culturelle de l'ERE, Gough préconise que les auteurs y intègrent un regard réflexif quant aux postulats théoriques qu'ils défendent.

4 Après avoir comparé le modèle de Kollmuss et Agyeman à celui proposé par Stem (2000), Phillip Payne (2002) soutient l'intérêt des travaux de Giddens en ERE et met l'emphase sur l'importance de cerner empiriquement certaines pratiques sociales, y incluant le rôle crucial que des habitudes, des routines et "autres conduites tacites " jouent dans la structuration du fonctionnement social. Cela, afin de poser un regard plus sociologique sur les conduites «environnementales ». Payne souligne également l'intérêt des travaux synthèses de Hart et Nolan et de Rickinson qui illustrent la 
transition des recherches actuelles en ERE vers des approches «interprétatives, critiques et post-modernes » de type ethnographique, ces positions épistémologiques reconnaissant les ancrages contextuels et culturels, valorisant les perspectives des sujets pour éclairer les logiques en jeu.

5 Pour terminer, retenons l'intérêt des idées avancées par Bjarne Bruun Jensen (2002) dans le dernier article de ce numéro spécial. Sur un mode pragmatique, il nous propose une reconceptualisation des concepts de «comportement pro-environnemental» (jugeant la proposition de départ trop limitée) et de «connaissance environnementale» afin de reconsidérer la soi-disant non-corrélation entre l'acquisition de connaissances environnementales et l'engagement social. Jensen soutient que la nature des connaissances scolaires habituelles sur l'environnement, tout comme la manière de les enseigner sur le mode d'une transmission passive, élude les processus d'appropriation effective par les élèves et ne constitue donc pas un enseignement orienté vers l'action et le changement social. Selon lui, il convient donc de rétablir des apprentissages sur l'environnement orientés explicitement vers l'engagement et la participation sociale des élèves. A cet effet, il propose une approche interdisciplinaire articulée autour de quatre axes complémentaires: une analyse à caractère scientifique de la question environnementale soulevée (on peut regretter cependant l'absence de problématisation des savoirs scientifiques); une analyse des causes structurelles du problème, à caractères social, économique et politique ; des connaissances quant aux stratégies de changement social envisageables (allant de l'analyse des relations de pouvoir en jeu à une réflexion sur les moyens à mettre en oeuvre en contexte pour modifier des pratiques); et enfin, une ouverture à d'autres manières de vivre en société. Une telle approche dont la mise en pratique semble bien documentée sur le plan empirique mériterait d'être considérée dans d'autres contextes scolaires.

6 Ce numéro de la revue Environmental Education Research illustre la vivacité des débats à caractère épistémologique dans ce champ de recherche. Le modèle de départ, à caractère instrumental, objet de discussion, est éclairé à partir de perspectives qui se réclament davantage du paradigme de la cognition située. La mise en résonance de ces deux types d'approches peut être féconde s'il s'agit de nourrir la réflexion de la communauté des chercheurs en ERE. Cependant, dès lors que l'on reconnaît les enjeux de pouvoir que transporte avec elle toute démarche de recherche, associée forcément à une certaine image de l'expertise et des savoirs des acteurs, l'instrumentalisation des conduites humaines et la non-inscription des positions théoriques des chercheurs dans une certaine représentation du contexte socio-historique en jeu devient intenable, que ce soit sur le plan de l'éthique de la recherche en ERE ou sur celui des enjeux sociopolitiques que transporte toute visée de changement social. Tenir compte de la recommandation de Hart (2000) en vue de renforcer la cohérence entre les orientations épistémologique, méthodologique (et j'ajouterais éthique), des orientations de recherche en ERE pour juger de leur pertinence en contexte, semble tout à fait d'actualité à la lecture des débats déployés ici. 


\section{BIBLIOGRAPHIE}

Gough, S. (2002). Whose gap ? Whose mind ? Plural rationalities and disappearing academics. Environmental Education Research, 8(3), 273-282.

Gough, S. et Scon, W. (1999). Education and training for sustainable tourism problems, possibilities and cautious First steps. Canadian Journal of Environmental Education. 4, 193-212.

Hart, P (2000). Requisite variety : the problem with generic guidelines for diverse genres of inquiry. Environmental Education Research, 2(1), 37-46.

Jensen, B.B. (2002). Knowledge, action and pro-environmental behaviour. Environmental Education Research, 8(3), 325-334.

Kollmuss, A. et Agyeman, J. (2002). Mind the gap : Why do people act environmentally and what are the barriers to pro-environmental behaviour? Environmental Education Research, 8(3), 239-260.

O'Donoghue, R. et Lotz-Sisidta, H. (2002). Some insights on the gap. Environmental Education Research, 8(3), 261-272.

Payne, P (2002). Post-metatheorizing environmental behaviours in environmental education. Environmental Education Research, 8(3), 307-314.

Stem, P (2000). Toward a coherent theory of environmentally significant behaviour. Journal of Social Issues, 56(3), 407-424.

\section{AUTEUR}

\section{BARBARA BADER}

Professeure de didactique des sciences, Université de Sherbrooke et chercheure associée au CIRADE 